\title{
Réponse à une sélection divergente sur la perte de poids des œufs de la caille japonaise en conservation
}

\author{
P Mérat, JL Monvoisin \\ Avec la collaboration technique de J Costa da Silva \\ Institut national de la recherche agronomique, \\ laboratoire de génétique factorielle, \\ 78352 Jouy-en-Josas Cedex France
}

(Reçu le 21 janvier 1991; accepté le 25 juin 1991)

Résumé - La sélection de 2 lignées divergentes de cailles japonaises, issues d'une origine commune, sur la perte de poids d'œufs non fécondés conservés $10 \mathrm{j}$ à $38^{\circ} \mathrm{C}$ a abouti après 9 générations à une perte double (en $\%$ du poids initial de l'œuf) dans la lignée «haute» comparée à la lignée «basse» : respectivement 14,8 et $7,2 \%$. L'héritabilité réalisée de la divergence entre lignées est de $0,14 \pm 0,02$.

Le poids moyen des œufs est plus élevé dans la lignée basse (de $2,1 \mathrm{~g}$ à la $9^{\mathrm{e}}$ génération) ainsi que le poids des cailleteaux à l'éclosion en $\%$ du poids initial de l'œuf. Corrélativement, le poids corporel reste plus élevé à 9 semaines dans la lignée basse, surtout chez les femelles. Toutefois, il n'apparaît pas de différence entre croisements réciproques, pour les cailleteaux femelles à $33 \mathrm{j}$, suggérant l'influence de facteurs autres que la perte de poids de l'œuf liés au génotype du descendant. Les lignées ne diffèrent ni pour la mortalité juvénile, ni pour le nombre d'œufs pondus contrôlés pour les premiers mois de production, ni pour l'épaisseur des coquilles. Le pourcentage d'œufs sans coquille ou à 2 jaunes est légèrement mais significativement plus élevé dans la lignée basse, et dans cette lignée, l'heure moyenne de ponte dans la journée est plus précoce. La consistance de l'albumen des œufs frais ou après conservation est plus élevée dans la lignée dont les œufs perdent moins de poids.

Les effets sur le taux d'éclosion n'ont pas été constants : à la $4^{\mathrm{e}}$ génération une forte baisse de ce taux a affecté la lignée «haute», alors qu'à la $9^{\mathrm{e}}$ génération, un avantage est associé à cette lignée et qu'il n'apparaît pas de différence significative entre lignées aux autres générations.

caille japonaise / sélection / œuf / perte de poids / porosité

Summary - Response to a divergent selection on the weight loss of unfertilized eggs after storage in the Japanese quail. The selection of two divergent lines of Japanese quails derived from a common origin on the loss of weight of unfertilized eggs stored 10 
days at $38^{\circ} \mathrm{C}$ resulted after 9 generutions in the high line having a double weight loss (as percent of the initial egg weight) in comparison with the low line: respectively 14.8 and $7.2 \%$. The realized heritability of the divergence between lines was $0.14 \pm 0.02$.

The mean egg weight was greater in the low line (by $2.1 \mathrm{~g}$ after 9 generations) and the same was observed for day-old body weight in percent of the initial egg weight. The mean body weight was still higher at 9 weeks of age in the low line, especially for females. However, no difference appeared between reciprocal crosses for the 33 week weight of the female progeny, suggesting the influence of factors other than egg weight loss, in relation with the genotype of the progeny. The lines did not differ for early mortality, egg production rate (short recording period after onset of laying) or for eggshell thickness. The percentage of shell-less and double-yolked eggs was slightly but significantly higher in the low line, and in this line the average time of lay in the day was earlier. The albumen quality of fresh or stored eggs was superior in the line with low egg weight loss. The effects on hatching rate were non consistent : at the 4 th generation a significant drop affected this rate in the high line, and in the 9th generation this line showed a better hatching percentage, but no significant difference between lines appeared in the other generations.

Japanese quail / selection / egg / weight loss / porosity

\section{INTRODUCTION}

La perte de poids des œufs conservés à température ambiante ou incubés correspond essentiellement à la perte d'eau par évaporation à travers les pores de la coquille. Divers auteurs ont décrit les modalités de cette perte d'eau et différentes méthodes d'évaluation de la porosité des coquilles (revues par Board et Scott, 1980; Tullet, 1984 par exemple). L'influence de différents facteurs a été passée en revue : poids des œufs, altitude (Tullett et Board, 1977), teneur en calcium de la ration (Tyler, 1945), âge de la poule (Tullett et Smith, 1983), position de l'œuf dans la série de ponte (Tyler, 1945).

L'intervention de facteurs génétiques a été mise en évidence sur la perte de poids de l'œuf ou la porosité de la coquille : effet d'une sélection pour la perte de poids de l'œuf chez la poule (Quinn et al, 1945); existence de différences entre lignées (Rauch, 1978, Scholtyssek et El-Bogdady, 1979; Arad et Marder, 1982; Gildersleeve, $1983)$; modification de cette perte de poids chez la caille après sélection sur le poids corporel (Pebbles et Marks, 1988).

Nous avons voulu dans l'expérience décrite ici, vérifier la possibilité chez la caille japonaise d'une sélection sur la perte de poids des œufs incubés à $38^{\circ} \mathrm{C}$ et les réponses liées éventuelles sur des variables intéressant la croissance, la production d'œufs ou la survie, qui, si elles existent pourraient avoir des conséquences pratiques.

\section{MATÉRIEL ET MÉTHODES}

\section{Animaux et reproduction}

En novembre 1983, un lot d'une population de base provenant du laboratoire de la faune sauvage de l'INRA, a été reproduit en groupe à partir de 60 mâles et 
120 femelles. Sur 51 filles issues de cette reproduction, la perte de poids de 5 œufs non fécondés par caille, estimée en pourcentage du poids initial des œufs après $10 \mathrm{j}$ d'incubation à $38^{\circ} \mathrm{C}$ et un degré hygrométrique d'environ $40 \%$, a servi de critère de sélection pour choisir les femelles de 16 couples de reproducteurs pour chacune des lignées projetées : les 16 femelles fondatrices de la lignée $p e^{+}$(«perte d'eau $+»)$ montraient la perte de poids la plus élevée, alors que les 16 femelles de la lignée «p $e^{-} »$ correspondaient aux valeurs individuelles les plus faibles. Les mâles étaient choisis au hasard. La reproduction de la première génération avait lieu en 2 éclosions en mars et avril 1984, correspondant chacune à une collecte des œufs sur 2 semaines. La sélection et la reproduction des générations suivantes s'effectuaient de manière semblable : choix individuel des femelles sur la perte de poids, dans les conditions décrites ci-dessus, d'œufs pondus par les femelles en contrôle à partir de l'âge de 9 semaines, choix des mâles dans les familles où des reproductrices avaient été gardées. Cependant, une mauvaise éclosion ayant été observée à la reproduction de la $4^{\mathrm{e}}$ génération dans la lignée $p e^{+}$, la sélection de cette lignée a été suspendue et remplacée par un tirage au hasard des parents entre les générations 4 et 5 .

Les accouplements étaient faits aléatoirement, sauf à éviter tout accouplement entre frères et sœurs ou demi-frères et demi-sœurs, a raison de 16 couples de parents par lignée.

Les reproductions des générations successives avaient lieu aux dates indiquées au tableau I.

En même temps que la $9^{\mathrm{e}}$ génération, les croisements réciproques entre les 2 lignées $\left(\sigma^{\top} p e^{+} \times \& p e^{-} ; \Im^{-} p e^{-} \times ? p e^{+}\right)$ont été reproduits à partir de 16 couples de parents, frères et sœurs de ceux ayant donné naissance aux lignées et éclos puis élevés en même temps que les descendants des lignées, auxquels ils ont été comparés.

\section{Conditions d'élevage - Variables mesurées}

Les cailleteaux des 2 lignées étaient élevés dans une même batterie chauffée jusqu'à l'âge de 4 semaines et demie, puis les femelles étaient placées en cages individuelles. Un éclairage artificiel était donné $14 \mathrm{~h} / 24 \mathrm{~h}$. Un aliment «jeunes» puis «pondeuses» standard était distribué ad libitum sous forme de farine.

Les variables mesurées sont indiquées dans les tableaux qui suivent. La variable «perte de poids de l'œuf», déjà définie, était mesurée sur le total de 5 œufs successifs par femelle, pondus à partir de l'âge de 9 semaines. Le poids moyen des mêmes œufs était enregistré avant leur mise en incubation. L'épaisseur de coquille était mesurée au même âge à la $5^{\mathrm{e}}$ génération seule. Cette mesure était faite avec un comparateur au 1/100mm à l'équateur de l'œuf, sur la coquille lavée et séchée, sans séparation de la membrane coquillière. Le taux de ponte (rapport du nombre d'œufs au nombre de j de contrôle), le pourcentage d'œufs sans coquille («mous»), à 2 jaunes ou cassés dans les cages, correspondaient à une période de contrôle de 1-4 mois suivant le mois d'entrée en ponte. Cette période n'a pu être maintenue constante à toutes les générations pour des raisons matérielles. Les 3 dernières variables citées ont été notées entre les générations 2 et 6 incluses.

À chaque génération, la valeur moyenne des lignées a été comparée par un test $t$, pour la variable sélectionnée et les autres paramètres mesurés. La régression 


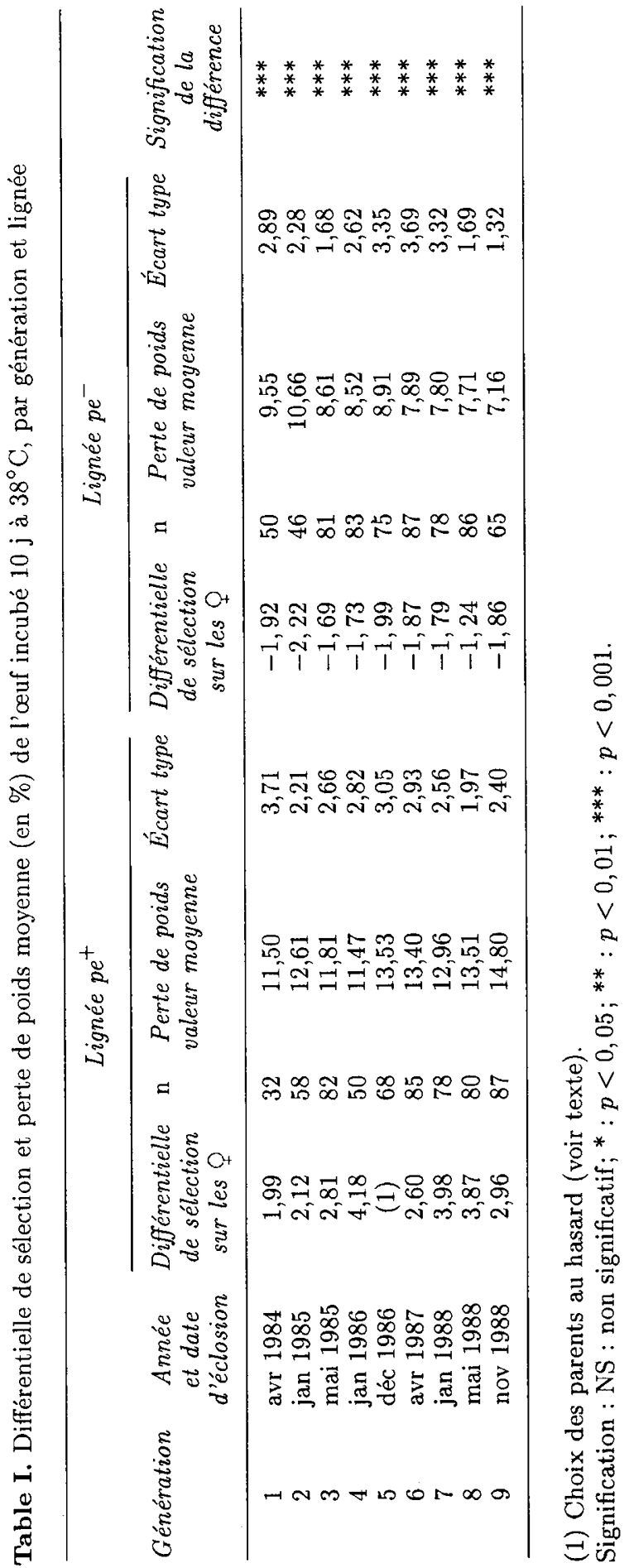


de la variable sélectionnée sur les générations et sa signification ont été estimées. Les corrélations phénotypiques entre les principales mesures ont été estimées à l'intérieur d'une même génération et d'une même lignée. Les effectifs n'ont pas été jugés suffisants pour permettre la présentation d'une estimation des corrélations génétiques.

\section{RÉSULTATS}

\section{Réponse à la sélection}

Le tableau I et la figure 1 montrent la réponse dans les 2 lignées aux générations successives pour le caractère sélectionné. Le tableau I indique en outre les intensités de sélection réalisées sur les femelles.

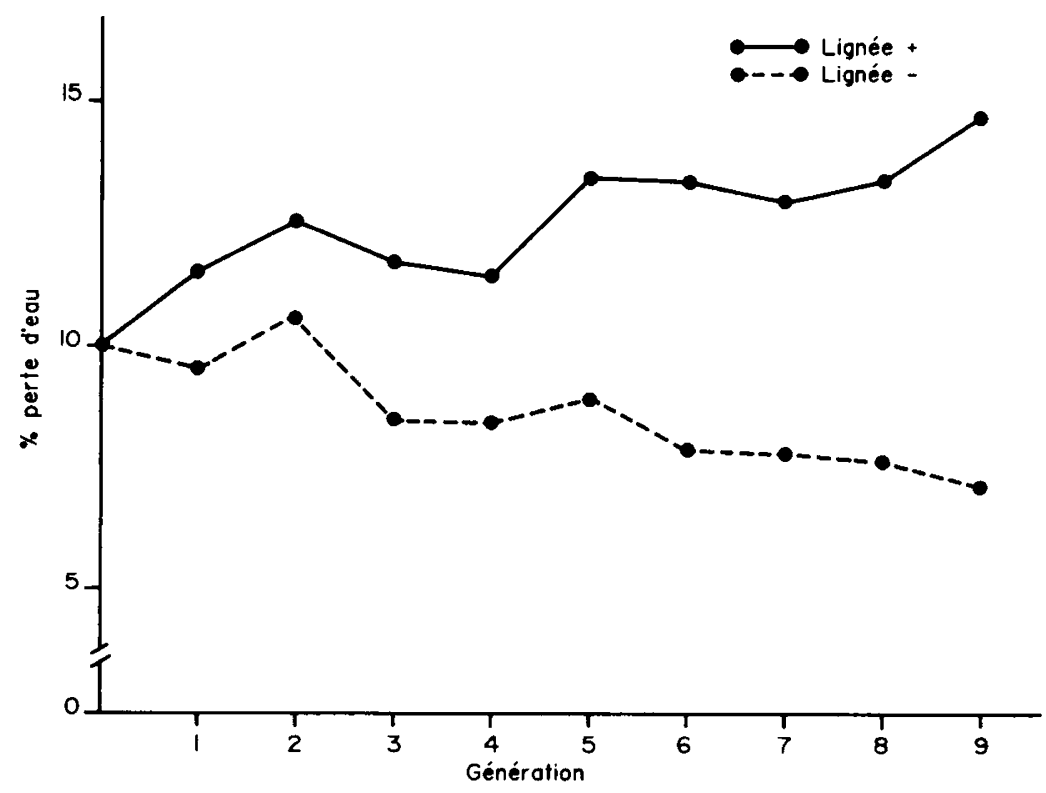

Fig 1. Évolution du pourcentage de perte d'eau de l'œuf au cours des générations de sélection.

Les 2 critères de sélection, valeur individuelle pour les femelles et valeur moyenne des sœurs attribuée aux mâles, sont supposés équivalents pour la prédiction des valeurs génétiques des candidats à la reproduction. L'évaluation des différentielles de sélection tient compte de la sélection exercée dans les 2 sexes. La reproduction, par couples, comportait le même nombre de parents mâles et femelles, et le taux de sélection exercé sur les mâles était d'ailleurs peu inférieur à celui exercé sur les femelles, à l'exception de la première génération, où les mâles étaient choisis au hasard. 
Compte tenu de ces remarques, l'héritabilité réalisée estimée jusqu'à la $9^{\text {e }}$ génération (régression de la divergence sur la différentielle de sélection cumulée) était $0,14 \pm 0,02$ pour l'ensemble des 2 lignées $(p<0,001)$, avec prise en compte de l'effet de la dérive génétique (Hill, 1972).

\section{Réponses liées}

Le tableau II présente, par génération, l'évolution pour chaque lignée du pourcentage d'éclosion, du poids moyen des œufs, du poids corporel dans chaque sexe à l'âge de 9 semaines et du taux de ponte pendant la période de contrôle.

Le tableau III donne les pourcentages d'œufs sans coquille (mous), cassés ou à 2 jaunes de la $2^{\mathrm{e}}$ à la $6^{\mathrm{e}}$ génération.

Au tableau IV figure, par lignées, la hauteur de l'albumen, mesure de sa consistance, pour des œufs frais ou après 1 ou 2 semaines de conservation sur la moyenne de 3 œufs par poule et par stade de conservation ( $9^{\mathrm{e}}$ génération). Le tableau $\mathrm{V}$ contient une comparaison des descendants de la $9^{\mathrm{e}}$ génération des lignées en sélection avec ceux issus de leurs croisements réciproques pour le taux d'éclosion, le rapport du poids du jeune à l'éclosion au poids de l'œuf avant incubation et le poids corporel à $33 \mathrm{j}$.

Le tableau VI montre qu'une augmentation du degré hygrométrique en incubation, réalisé sur une éclosion supplémentaire aux générations 7 et 8 , ne modifie pas le taux d'éclosion dans l'une au l'autre lignée.

Les variables suivantes, dont certaines ont été mesurées seulement occasionnellement, ne sont pas comprises dans les tableaux précédents :

- la mortalité des jeunes jusqu'à l'âge de 4 semaines et demie ne différait pas significativement entre lignées au total (pourcentage moyen sur l'ensemble des générations : 20,4\% sur 1909 animaux éclos dans la lignée $p e^{+} ; 18,2 \%$ sur 1792 oiseaux éclos dans la lignée $p e^{-}$).

- l'épaisseur des coquilles, mesurée à la $5^{\mathrm{e}}$ génération sur 1 œuf par femelle, avait pour valeur moyenne (en 1/100 mm) 20,3 et 20,1 respectivement sur 51 œufs de la lignée $p e^{+}$et 61 œufs de la lignée $p e^{-}$; différence non significative.

- le pourcentage d'œufs pondus avant $10 \mathrm{~h}$ du matin évalué à la $9^{\text {e }}$ génération, respectivement sur 1292 et 772 œufs des lignées $p e^{+}$et $p e^{-}$sur une durée totale de 2 semaines, était de $48,2 \%$ pour la première et $56,7 \%$ pour la seconde $(p<0,001)$. La lignée $p e^{-}$apparaît comme pondant plus tôt en moyenne dans la journée.

Parallèlement à la recherche de réponses liées, les corrélations phénotypiques intralignées et générations ont été calculées entre le caractère sélectionné, les poids corporels et le poids moyen des œufs. Ces corrélations ont été trouvées au total faibles et non significatives (peut-être du fait de la variation limitée du caractère principal intralignées) et ne sont pas commentées plus avant.

\section{DISCUSSION ET CONCLUSIONS}

La réponse à la sélection sur la perte de poids de l'œuf a, selon les apparences, été effective dans les 2 sens. Elle a abouti au bout de 9 générations à une perte double (en pourcentage du poids initial de l'œuf) dans la lignée $p e^{+}$comparée à 
la lignée $p e^{-}$. Par rapport à la moyenne de la population de départ, la lignée $p e^{+}$ a réalisé une augmentation de perte de poids de l'œuf de $39,2 \%$ et la lignée peune diminution de $32,5 \%$ : par rapport à la valeur moyenne de départ $(10,6 \%)$, la divergence est de $71,7 \%$.

Une réponse liée très marquée concerne le poids moyen des œufs, supérieur de $18,7 \%$ dans la lignée $p e^{-}$comparée à $p e^{+}$à la $9^{\text {e }}$ génération de sélection. La différence entre lignées pour cette variable apparaît à la $3^{\mathrm{e}}$ génération, et l'évolution de la valeur moyenne dans chaque lignée suggère d'autre part que le poids de l'œuf a augmenté dans la lignée $p e^{-}$tout en restant à peu près inchangé dans la lignée à perte de poids élevée.

Le poids de l'œuf par lui-même peut influer sur la perte de poids en incubation à perméabilité égale par unité de surface, un œuf de plus grande taille ayant une surface de coquille relativement moindre qu'un petit œuf. Cependant, en admettant que la surface augmente comme la puissance $2 / 3$ du volume ou du poids, la différence de poids d'œuf entre les 2 lignées à la $9^{\mathrm{e}}$ génération, représentant $19 \%$ du poids d'œuf moyen de la lignée $p e^{+}$, fait prévoir une différence de perte de poids de l'ordre de $6 \%$, alors qu'à cette génération la perte moyenne observée dans la lignée $p e^{+}$atteint plus du double de celle de la lignée $p e^{-}$, soit $100 \%$ de plus. Si la considération du rapport surface/volume de l'œuf peut probablement rendre compte de la réponse liée à la sélection en ce qui concerne le poids moyen des œufs, par contre la variation observée de ce poids moyen au cours des générations ne peut à elle seule être la cause de l'écart obtenu entre lignées pour la perte de poids des œufs incubés. Cette dernière doit correspondre à une différence dans le nombre ou le diamètre moyen des pores de la coquille.

L'effet sur le poids des œufs peut expliquer au moins en partie la valeur plus élevée du poids corporel à 9 semaines dans la lignée $p e^{-}$. Cette augmentation est plus nette chez les femelles où elle est de l'ordre de $6 \%$, quoique, dans l'ensemble, une tendance de même sens apparaisse chez les mâles. Ce résultat peut être rapproché de l'effet trouvé sur la perte de poids des œufs par Pebbles et Marks (1988) à la suite d'une sélection sur le poids corporel à 4 semaines. Le poids du cailleteau d'1 j rapporté au poids de l'œuf est aussi trouvé plus élevé dans la lignée pe- que dans $p e^{+}($tableau V). Il en est de même dans le croisement dont la mère est issue de la ligne $p e^{-}$par comparaison au croisement réciproque, mais de façon plus accentuée. Par contre, ces croisements réciproques ne montrent pas de différence nette pour le poids à $33 \mathrm{j}$ des femelles, suggérant que pour ce caractère, l'effet du génotype du zygote joue un rôle parallèlement à l'effet prolongé du poids et de la perte du poids de l'œuf.

Dans l'expérience présente, l'épaisseur et la solidité des coquilles (exprimée par le pourcentage d'œufs cassés) ne sont pas affectés par la sélection sur la perte de poids.

La valeur plus élevée de la hauteur de l'albumen (tableau IV) dans la lignée $p e^{-}$ peut s'interpréter par une perméabilité moindre de la coquille dans cette lignée, non seulement à la vapeur d'eau mais aussi au gaz carbonique dont on sait qu'il conditionne la consistance de l'albumen (Sauveur, 1988). Par contre, on ne trouve pas d'effet constant de la sélection sur le taux d'éclosion; à la $4^{\mathrm{e}}$ génération, la lignée $p e^{+}$montre un taux d'éclosion inférieur, à la $9^{\mathrm{e}}$ génération, sa valeur est 
Tableau II. Comparaison des lignées intra-génération pour le taux d'éclosion, le poids moyen des œufs, le poids corporel et la ponte.

\begin{tabular}{|c|c|c|c|c|c|c|c|c|c|c|c|c|}
\hline \multirow[b]{2}{*}{$\begin{array}{l}\text { Géné- } \\
\text { ration }\end{array}$} & \multicolumn{5}{|c|}{$\begin{array}{c}\text { Taux d'éclosion } \\
\text { (cailleteaux nés/œeufs incubés \%) }\end{array}$} & \multicolumn{7}{|c|}{ Poids moyen des aufs à 9 semaines } \\
\hline & ${ }_{\mathrm{N}}^{p e}$ & $\begin{array}{l}\% \\
\text { éclos }\end{array}$ & ${ }_{\mathrm{N}}^{p e}$ & $\begin{array}{l}\% \\
\text { éclos }\end{array}$ & $\begin{array}{l}\text { Signifi- } \\
\text { cation }\end{array}$ & $\mathrm{N}$ & $\begin{array}{c}p e^{+} \\
\text {valeur } \\
\text { moyenne }\end{array}$ & $\mathrm{N}$ & $\begin{array}{c}p e^{-} \\
\text {valeur } \\
\text { moyenne }\end{array}$ & $e^{\begin{array}{c}\text { Signifi- } \\
\text { cation }\end{array}}$ & $\mathrm{N}$ & $\begin{array}{l}\text { pe }^{+} \\
\text {valeur } \\
\text { moyenne }\end{array}$ \\
\hline 1 & 283 & 63,2 & 273 & 65,6 & NS & 28 & 11,15 & 43 & 11,44 & NS & - & - \\
\hline 2 & 292 & 53,4 & 233 & 47,6 & NS & 50 & 11,33 & 37 & 11,86 & NS & - & - \\
\hline 3 & 361 & 71,4 & 343 & 73,8 & NS & 78 & 11,81 & 76 & 12,77 & $* * *$ & 88 & 180,3 \\
\hline 4 & 434 & 44,3 & 400 & 68,1 & $* * *$ & 51 & 12,32 & 84 & 13,03 & $* * *$ & - & - \\
\hline 5 & 404 & 61,4 & 338 & 60,1 & NS & 65 & 11,88 & 66 & 13,28 & $* * *$ & 95 & 166,3 \\
\hline 6 & 374 & 76,0 & 330 & 72,3 & NS & 82 & 12,10 & 74 & 13,46 & $* * *$ & 85 & 183,0 \\
\hline 7 & 342 & 63,5 & 323 & 57,4 & NS & 83 & 12,06 & 89 & 13,04 & $* * *$ & 91 & 180,2 \\
\hline 8 & 346 & 61,8 & 387 & 59,4 & NS & 80 & 11,51 & 86 & 12,72 & $* * *$ & 97 & 182,1 \\
\hline 9 & 396 & 65,9 & 317 & 55,8 & $* *$ & 87 & 11,29 & 65 & 13,40 & $* * *$ & 104 & 179,8 \\
\hline
\end{tabular}

1 Signification : voir tableau I.

supérieure à celle de la lignée $p e^{-}$, et les autres comparaisons ne montrent pas de différence, ce qui peut suggérer une réponse différente des 2 lignées à des facteurs non contrôlés de l'environnement intervenant de façon occasionnelle, et s'accorde par ailleurs avec l'observation générale selon laquelle une variation dans des limites assez larges de la teneur en eau de l'œuf ou de l'embryon est sans influence sur le taux d'éclosion (Decuypere et al, 1990). L'absence de différence entre lignées pour la mortalité du jeune âge peut aller dans le même sens.

La ponte ne diffère pas entre lignées, du moins pour la période de contrôle, limitée qui a pu être faite. D'autre part, l'heure de ponte plus précoce au cours de la journée dans la lignée $p e^{-}$et le pourcentage un peu plus élevé d'œufs sans coquille ou à 2 jaunes qu'elle présente, peuvent s'accorder avec l'hypothèse d'une durée de formation de l'œuf plus courte dans cette lignée. Nous n'avons pas connaissance d'une relation directe trouvée antérieurement entre cette durée de formation et la perte de poids des œufs. Cependant, Tyler (1945) note que les premiers œufs d'une série de ponte (pondus plus tôt) chez la poule sont moins «poreux» que les suivants. Chez la poule également, on a trouvé une relation entre la position de l'œuf dans la série ou l'heure de ponte et l'épaisseur de coquille, les œufs pondus plus tard dans la journée ayant en moyenne des coquilles plus épaisses (Sauveur, 1988; Belyavin et al, 1989). Dans le cas présent, la lignée pondant des œufs plus tôt en moyenne $\left(p e^{-}\right)$n'a pas une moindre épaisseur de coquille, ce qui suggère un dépôt légèrement plus important de calcium par unité de temps. 
Tableau II (suite)

Poids corporel à 9 semaines

Pourcentage de ponte

des survivantes

\begin{tabular}{|c|c|c|c|c|c|c|c|c|c|c|c|c|}
\hline $\mathrm{N}$ & $\begin{array}{l}p e^{-} \\
\text {valeur } \\
\text { moyenne }\end{array}$ & $\begin{array}{l}\text { Signifi- } \\
\text { cation }\end{array}$ & $\mathrm{N}$ & $\begin{array}{l}\text { pet } \\
\text { valeur } \\
\text { moyenne }\end{array}$ & $\mathrm{N}$ & $\begin{array}{l}p e^{-} \\
\text {valeur } \\
\text { moyenne }\end{array}$ & $\begin{array}{l}\text { Signifi- } \\
\text { cation }\end{array}$ & $\mathrm{N}$ & $\begin{array}{l}p e^{+} \\
\text {valeur } \\
\text { moyenne }\end{array}$ & $\mathrm{N}$ & $\begin{array}{l}p e^{-} \\
\text {valeur } \\
\text { moyenne }\end{array}$ & $\begin{array}{l}\text { Signifi- } \\
\text { cation }\end{array}$ \\
\hline- & - & - & - & - & - & - & - & - & - & - & - & - \\
\hline- & - & - & - & - & - & - & - & 54 & 61,2 & 43 & 56,3 & NS \\
\hline 114 & 185,7 & $*$ & 66 & 216,5 & 100 & 235,3 & $* *$ & 85 & 82,3 & 81 & 74,1 & NS \\
\hline- & - & - & - & - & - & - & - & 55 & 76,4 & 89 & 77,8 & NS \\
\hline 77 & 181,2 & * & 72 & 214,2 & 80 & 226,0 & $* *$ & 65 & 85,9 & 74 & 86,6 & NS \\
\hline 97 & 179,8 & NS & 103 & 206,2 & 107 & 217,4 & $* * *$ & 88 & 83,1 & 94 & 83,9 & NS \\
\hline 94 & 182,0 & NS & 92 & 209,7 & 89 & 224,9 & $* * *$ & 90 & 74,9 & 84 & 55,9 & $* *$ \\
\hline 91 & 185,7 & NS & 88 & 206,7 & 97 & 215,2 & $* *$ & 83 & 61,9 & 87 & 63,0 & NS \\
\hline 78 & 184,7 & $*$ & 116 & 208,6 & 63 & 215,8 & $* *$ & 111 & 63,0 & 65 & 64,9 & NS \\
\hline
\end{tabular}

Tableau III. Pourcentage d'œufs «mous», cassés ou à 2 jaunes par génération et au total dans les 2 lignées $p e^{+}$et $p e^{-}$

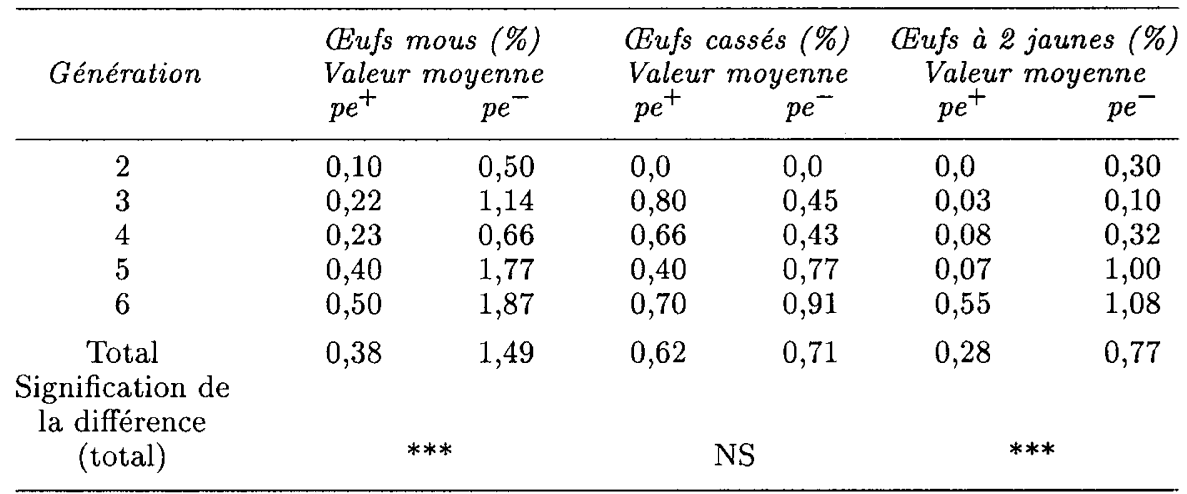

Signification : voir tableau I.

Tableau IV. Hauteur de l'albumen de l'œuf frais ou après conservation pour les lignées $p e^{+}$et $p e^{-}\left(9^{\mathrm{e}}\right.$ génération $)$.

\begin{tabular}{|c|c|c|c|c|c|c|}
\hline & \multicolumn{6}{|c|}{ Durée de conservation } \\
\hline & \multicolumn{2}{|c|}{$1 j$} & \multicolumn{2}{|c|}{$8 j$} & \multicolumn{2}{|c|}{$15 j$} \\
\hline & $p e^{+}$ & $p e^{-}$ & $p e^{+}$ & $p e^{-}$ & $p e^{+}$ & $p e^{-}$ \\
\hline Nombre de poules & 34 & 30 & 34 & 30 & 34 & 30 \\
\hline Valeur moyenne $(0,1 \mathrm{~mm})$ & 51,6 & 57,3 & 38,7 & 42,4 & 32,0 & 35,8 \\
\hline$t$ et signification & \multicolumn{2}{|c|}{$3,45^{* * *}$} & \multicolumn{2}{|c|}{$2,08^{*}$} & \multicolumn{2}{|c|}{$2,69 * *$} \\
\hline
\end{tabular}

Signification : voir tableau I. 
Tableau VI. Incubation des lignées $p e^{+}$et $p e^{-}$avec un taux hygrométrique élevé ou non.

\begin{tabular}{|c|c|c|c|c|c|}
\hline \multirow{3}{*}{$\begin{array}{l}\text { Génération et taux } \\
\text { hygrométrique }\end{array}$} & \multicolumn{4}{|c|}{ Lignée } & \multirow{3}{*}{$\begin{array}{c}\text { Signification de } \\
\text { la différence } \\
p e^{+} p e^{-}\end{array}$} \\
\hline & \multicolumn{2}{|r|}{$p e^{+}$} & \multicolumn{2}{|r|}{$p e^{-}$} & \\
\hline & $N$ & Éclos/incubés \% & $\mathrm{N}$ & Éclos/Incubés \% & \\
\hline \multicolumn{6}{|l|}{ re génération } \\
\hline $\begin{array}{l}\text { Hygrométrie élevée } \\
\text { (valeur moyenne } 70 \% \text { ) }\end{array}$ & 172 & 66,3 & 184 & 56,4 & NS \\
\hline $\begin{array}{l}\text { Hygrométrie basse } \\
\text { (valeur moyenne } 45 \% \text { ) }\end{array}$ & 342 & 63,5 & 323 & 57,4 & NS \\
\hline \multicolumn{6}{|l|}{$8^{e}$ génération } \\
\hline Hygrométrie élevée & 204 & 55,4 & 212 & 54,3 & NS \\
\hline Hygrométrie basse & 346 & 61,8 & 387 & 59,4 & NS \\
\hline
\end{tabular}

Signification : voir tableau I.

\section{RÉFÉRENCES}

Arad Z, Marder J (1982) Egg-shell water vapor conductance of the domestic fowl: comparison between two breeds and their crosses. Br Poult Sci 59, 325-328

Belyavin CG, Boorman KN, Volynchook J (1989) Egg quality in individual birds. In: Egg quality: current problems and recent advances (RG Wells et CG Belyavin, eds) Butterworths, Londres, chapitre 7

Board RG, Scott VD (1980) Porosity of the avian eggshell. Am Zool 20, 339-349

Decuypere E, Dewil E, Kühn ER (1990) The hatching process and the role of hormones. Br Poult Sci Symp 22, 239-256

Gildersleeve RP (1983) The effect of humidity and broiler strain on egg weight losses during incubation. Poult Sci 62, 1428 (abstr)

Hill WG (1972) Estimation of realized heritabilities from selection experiments. I. Divergent selection. Biometrics 28, 747-765

Pebbles ED, Marks HL (1988) Incubation and weight loss and embryonic development in Japanese quail selected under different dietary environments. Poult Sci 67 suppl 1 , (abstr), 28

Quinn JP, Gordon CD, Godfrey AB (1945) Breeding for egg-shell quality as indicated by egg weight loss. Poult Sci 24, 399-403

Rauch HW (1978) Veränderung der Eilkar und Luftkammerhöle von weissen und braunen Eltern nach 4-und 8-wöchiger Lagerung. $16^{e}$ Congrès Mondial d'Aviculture, 976-983

Sauveur B (1988) Qualité de l'œuf. In: chapitre 14 Reproduction des volailles et production d'œufs. INRA Paris, p 403; p 414

Scholtyssek S, El-Bogdagy A (1979) Herkunfts- und Lagerungseinflüsse auf die innere Eiqualität, insbesondere die Proteine im dünnen und dicken Eiklar. Arch Geflügelkde $43,245-252$

Tullett SG (1984) The porosity of avian eggshells. Comp Biochem Physiol 78A, 5-13 
Tullet SG, Board RG (1977) Determinants of avian eggshell porosity. J Zool (Lond) 183, 203-211

Tullet SG, Smith S (1983) A note on changes in eggshell porosity with flock age and season during the first breeding cycle of domestic chicks. Br Poult Sci 24, 501-509 Tyler C (1945) The porosity of eggshells, and the influence of different levels of dietary calcium upon porosity. J Agric Sci 35, 168-177 